\title{
IDENTIFYING THE MOTOR NEURON DISEASE IN EMG SIGNAL USING TIME AND FREQUENCY DOMAIN FEATURES WITH COMPARISON
}

\author{
${ }^{1}$ Shaikh Anowarul Fattah, ${ }^{2} \mathrm{Md}$. Asif Iqbal, ${ }^{2}$ Marzuka Ahmed Jumana, \\ and ${ }^{2}$ A. B. M. Sayeed Ud Doulah \\ ${ }^{1,2}$ Department of EEE, Bangladesh University of Engineering and Technology, Dhaka \\ Bangladesh \\ fattah@eee. buet.ac.bd \\ ${ }^{2}$ Department of EECE, Military Institute of Science and Technology (MIST), Dhaka \\ Bangladesh \\ asif_eece_mist@yahoo.com, jumana_eece5@yahoo.com, \\ sayeed.doulah@gmail.com
}

\begin{abstract}
Motor neuron diseases are the most common neurological disorders found in the age ranges between 35-70 years, which selectively affect the motor neurons. Amyotrophic lateral sclerosis (ALS) is a fatal motor neuron disease that assails the nerve cells in the brain. This disease progressively degenerates the motor cells in the brain and spinal cord, which are responsible for controlling the muscles that enable human to move around, breathe, speak, and swallow. The electromyography (EMG) signals are the biomedical signals that are used to study the muscle function based on the electrical signal originated from the muscles. As the nervous system controls the muscle activity, the EMG signals can be viewed and analyzed in order to detect the indispensable features of the ALS disease in individuals. In this paper, analyzing the time and frequency domain behaviour of the EMG signals obtained from several normal persons and the ALS patients, some characteristic features, such as autocorrelation, zero crossing rate and Fourier transform are proposed to identify the ALS disease. For the pupose of classification, K-nearest neighbothood classifier is employed in a leave-one out cross validation technique. In order to show the classification performance, an EMG database consisted of 7 normal subjects aged 21-37 years and 6 ALS patients aged 35-67 years is considered and it is found that the proposed method is capable of distinctly separating the ALS patients from the normal persons.
\end{abstract}

\section{KEYWORDS}

Amyotrophic lateral sclerosis (ALS), electromyography (EMG), autocorrelation, zero crossing rate, Fourier transform, KNN classifier.

\section{INTRODUCTION}

The electromyography (EMG) signal is a biomedical signal that is obtained via electrical response generated in muscles during its contraction representing neuromuscular activities. The muscle activity (contraction/relaxation) is always controlled by the nervous system. The EMG signal exhibits complicated characteristics since it is dependent on the anatomical and physiological properties of muscles and controlled by the nervous system. However, it serves as a reliable source of information about different features of muscle function [1-3]. A good understanding of the EMG signal can lead to successful clinical diagnosis for different biomedical applications. One of the important application areas is the identification of motor disability. The structural unit 
of contraction is the muscle fibre. An EMG signal is the train of motor unit action potential (MUAP). The shapes and firing rates of MUAPs in EMG signals render significant source of information for the diagnosis of neuromuscular disorders.

Surface EMG (sEMG) is a method of recording the information present in the muscle action potentials. This sEMG signal can be measured by employing conductive elements or electrodes on the skin surface. In the process of acquiring sEMG signal from the electrodes mounted directly on the skin, it is found that the signal consists of all the MUAPs occurring in the muscles. As these action potentials occur at random intervals, the generated voltage corresponding to the EMG signal may be either positive or negative. The EMG signal can also be acquired invasively by inserting the wire or needle electrodes directly in the muscle. Combination of the muscle fiber action potentials generated from all the muscle fibers of a single motor unit, namely the MUAP, can be detected by a skin surface electrode (non-invasive) placed near this field, or by a needle electrode (invasive) inserted in the muscle [4]. In view of analyzing the EMG signal, generally it is first picked up from the electrodes, amplified using differential amplifiers and then preprocessed to eliminate low- and high-frequency noises and possible artifacts. Finally, the noisereduced signal is rectified and averaged in some format to indicate the EMG amplitude. Surface EMG is the more common method of measurement, since it is non-invasive and can be conducted by personnel other than physicians with minimal risk to the subject. Measurement of sEMG is dependent on a number of factors and its amplitude varies from the microvolt to a low millivolt range [1]. The time and frequency domain properties of the sEMG signal depend on different factors, such as the timing and intensity of muscle contraction, the distance of the electrode from the active muscle area, the properties of the overlying tissue (e.g. thickness of overlying skin and adipose tissue), the electrode and amplifier properties and the quality of contact between the electrode and the skin [5-9].

The amytrophic lateral sclerosis (ALS) is the most common variant of motor neuron diseases. It is also known as Lou Gehrig's disease (after Lou Gehrig, a famous baseball player who was diagnosed with ALS in 1939). It is a progressive neurodegenerative disorder that affects both the upper and lower motor neurons. Motor neurons are nerve cells that control muscle movement. Upper motor neurons send messages from the brain to the spinal cord and lower motor neurons send messages from the spinal cord to the muscles. Hence the motor neurons are the most important part of the body's neuromuscular system. The ALS disease damages motor neurons in the brain and spinal cord. It causes these motor neurons to shrink and disappear, so that the muscles no longer receive signals to move. As a result, the muscles become smaller and weaker. Gradually the body becomes paralyzed, which means that the muscles no longer work [10]. The ALS can occur among young individuals, but it most commonly affects people between the ages of 35-70, with a slight male predominance. It is difficult to diagnose in the early stages because its symptoms may mimic other disorders. However, there are some clinical signs which may be treated as indication of damages either in the upper or in the lower motor neurons. A lower motor neuron lesion is characterized by muscle atrophy, weakness, fasciculation and cramps.

One possible way to determine the existence of the ALS is to analyze the EMG signal. In order to observe the effect of ALS on the recorded EMG signal, in most of the cases, changes in values of some selected EMG parameters are monitored and these individual parameters are achieved as a consequence of processing the EMG signal in time and frequency domains [11-15]. The objective of this paper is to develop a method to classify the ALS patients and the normal persons based on distinguishable characteristic features of the EMG signal. In this respect, some time and frequency domain features of the EMG signal are proposed with detailed experimental validation considering some standard EMG databases.

The paper is organized as follows. First, a brief description of the EMG signal and a preliminary idea about the ALS disease are presented. Next, proposed features of the EMG signal and an 
Signal \& Image Processing : An International Journal (SIPIJ) Vol.3, No.2, April 2012

analysis of the robustness of those features are shown. Finally, classification between the normal control group and the ALS patients has been carried out using the proposed method.

\section{MATERIAL AND MeTHODS}

\subsection{Experimental Dataset}

The experimental dataset is consisted of a normal control group and a group of patients with the ALS. In the control group, there are 6 normal subjects, 3 females and 3 males, all within the age limit 21-37 years. All of them are in general good physical shape and none had signs or history of neuromuscular disorders. The ALS patient group is consisted of 6 patients, 2 females and 4 males aged 35-67 years. Besides clinical and electrophysiological signs compatible with the ALS, 4 of them died within a few years after onset of the disorder, supporting the diagnosis of the ALS. The brachial biceps muscles were used in this study because they were the most frequently investigated in the two patient groups. During the recording of the EMG signals, following conventional conditions for MUAP analysis were maintained: (1) the recordings were made at low (just above threshold) voluntary and constant level of contraction, (2) visual and audio feedback were used to monitor the signal quality, (3) a standard concentric needle electrode was used, (4) the EMG signals were recorded from five places in the muscle at three levels of insertion (deep, medium, low), and (5) the high and low pass filters of the EMG amplifier were set at $2 \mathrm{~Hz}$ and $10 \mathrm{kHz}[16]$.

\subsection{Time and Frequency Domain Features of EMG Signals}

Because of the complicated nature of the EMG signals, it would not be a convincing approach to classify them directly based on the time variation of the data as observed. The variation in data pattern of the EMG signals obtained from a normal person and an ALS patient is generally not uniquely distinguishable. As a result, further detailed analysis using both temporal and spectral representations would be definitely helpful in EMG data classification. It is well known that different time and frequency domain analyses turn out to be very effective for the analysis of transient signals [17-18]. Considering the computational simplicity and well acceptance in clinical practice, in this paper, fast Fourier transform (FFT) is used to obtain frequency domain features and for time domain characteristics, autocorrelation and zero crossing rates are utilized.

\subsubsection{Spectral feature}

In order to investigate the spectral characteristics of the EMG signal, in the proposed method, only magnitude spectrum of the EMG signal is taken into consideration. Especial attention has been given on some specific spectral characteristics, such as spectral energy distribution pattern at different frequencies, tendency of concentrating maximum energy at any particular frequency, and average and peak spectral amplitude and frequency. For the purpose of spectral analysis, short time Fourier transform is employed, which is most widely used for the data analysis in areas, such as biomedical signal and image processing [19], [20]. In particular, the fast Fourier transform (FFT) is used for determining the magnitude spectrum of the EMG signal. It is expected that within a short duration of the EMG data, the spectral behaviour remains consistent. Hence from a long duration of the EMG recording, for short time spectral analysis, smaller frames are extracted by using windowing techniques. However, effect of windowing in time domain may generate unwanted ripples in spectral domain.

In Figs. 1 and 2, the FFT magnitude spectra of the frame of EMG data for the first and last three normal persons are shown, respectively. For each person, five different frames are chosen arbitrarily. In a similar fashion, in Figs. 3 and 4, the FFT magnitude spectra of the frame of EMG data for the first and last three ALS patients are shown, respectively. 
Signal \& Image Processing : An International Journal (SIPIJ) Vol.3, No.2, April 2012

\section{FFT of 5 Arbitrary Frames of 3 Normal Persons}
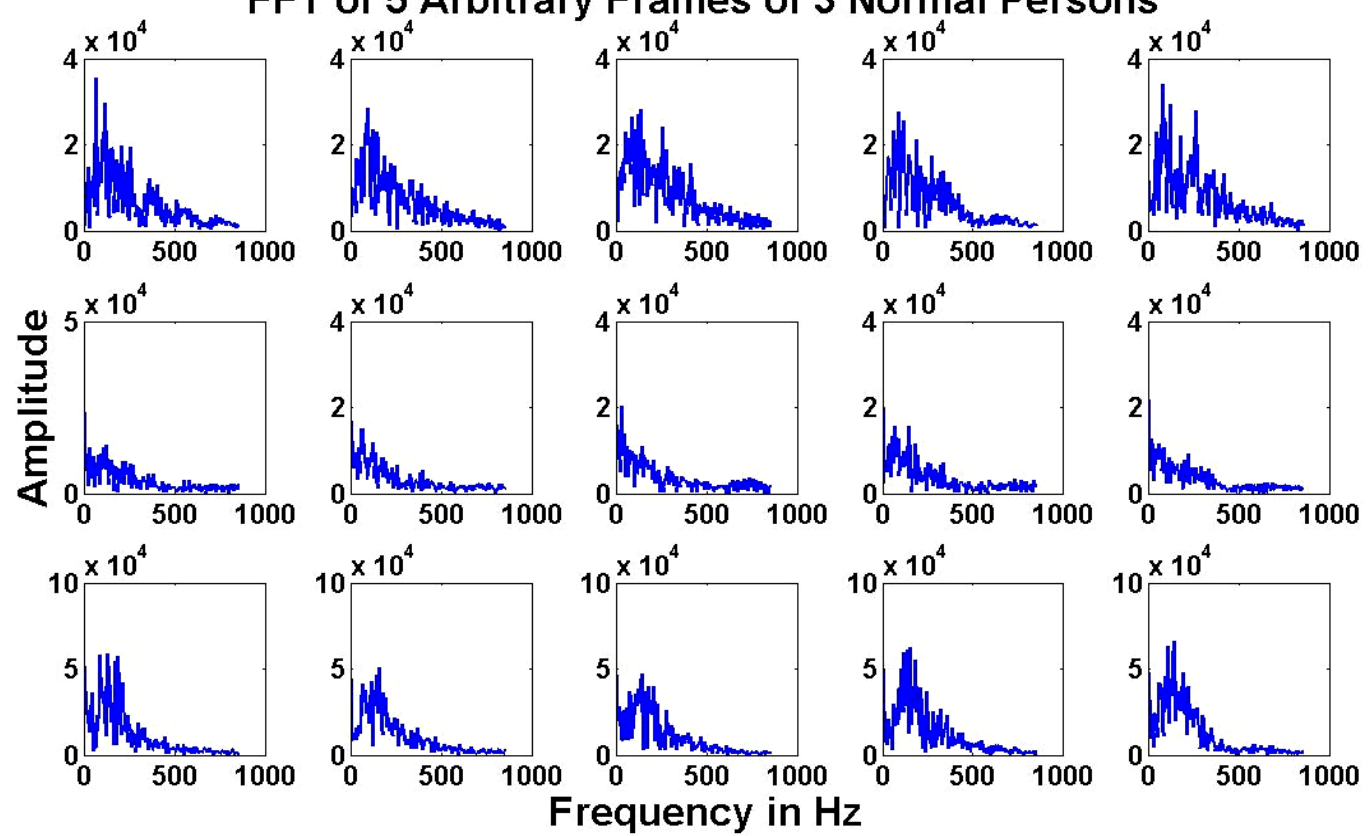

Frequency in $\mathrm{Hz}$

Figure 1. Magnitude spectra of the frame of EMG data for the first three normal persons.
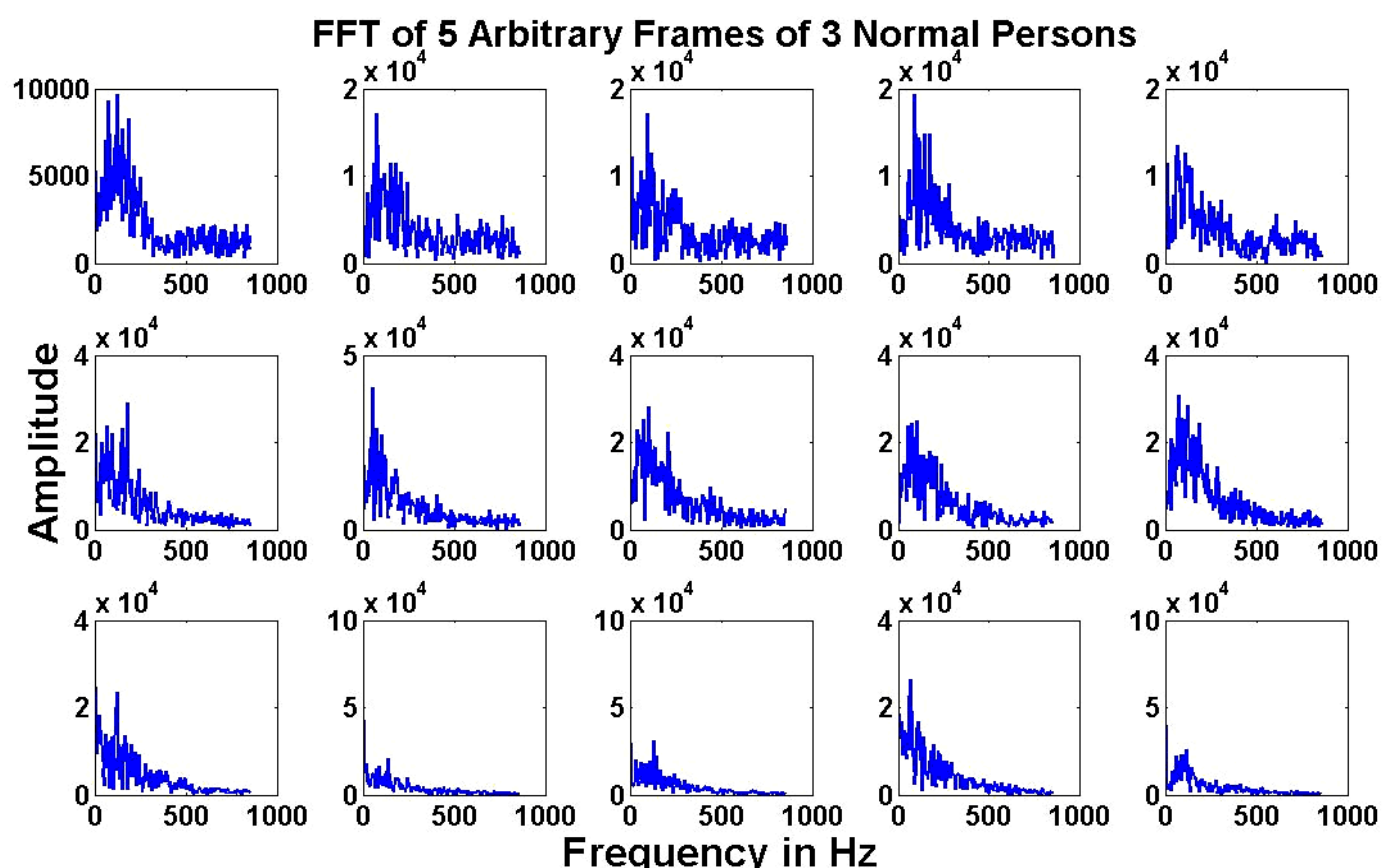

Figure 2. Magnitude spectra of the frame of EMG data for the last three normal persons. 
Signal \& Image Processing : An International Journal (SIPIJ) Vol.3, No.2, April 2012

It is observed from the above figures that the height of the magnitude spectra for the case of ALS patients is comparatively higher than that obtained for the normal persons. It is also evident from magnitude spectra that spectral energy is mostly concentrated in the low frequency
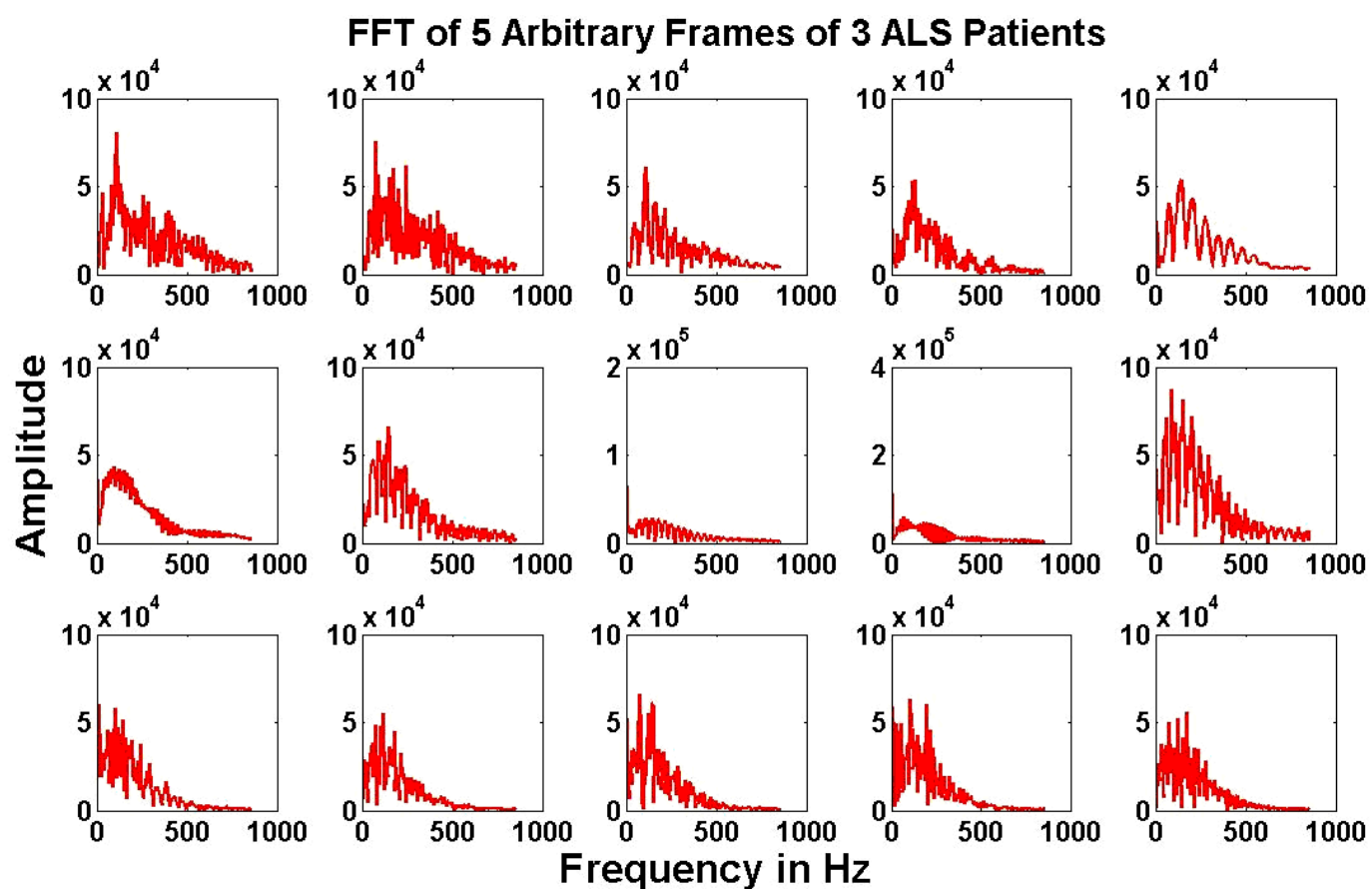

Figure 3. Magnitude spectra of the frame of EMG data for the first three ALS patients.
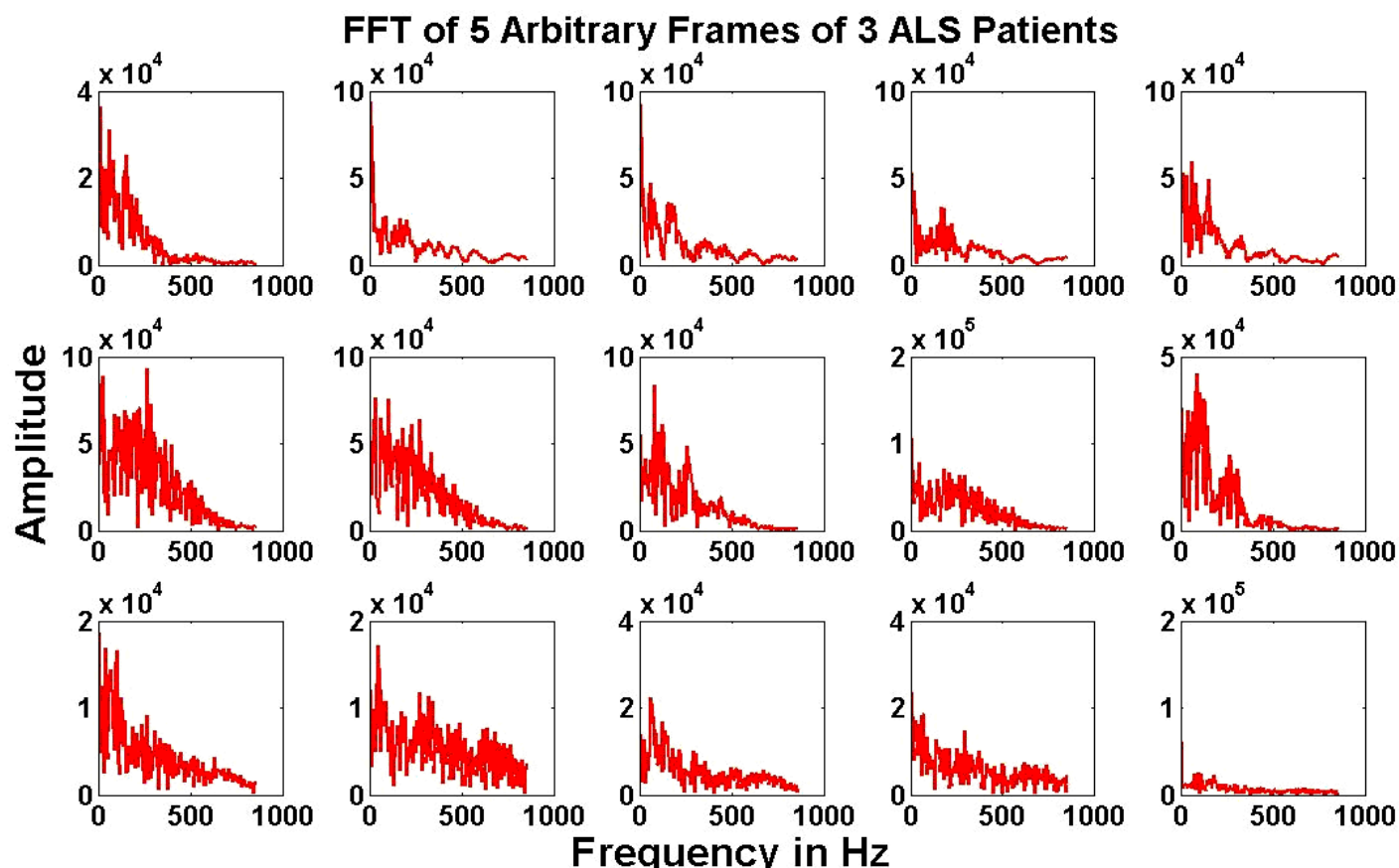

Figure 4. Magnitude spectra of the frame of EMG data for the last three ALS patients. 
Signal \& Image Processing : An International Journal (SIPIJ) Vol.3, No.2, April 2012

regions for both normal persons and ALS patients. Maximum peaks of the magnitude spectra for different cases appear at different frequency locations at a random fashion. As a result, instead of considering the frequency values of the maximum spectral peaks, only their amplitude values are taken into consideration as a distinguishing spectral feature.

Table 1. Maximum, minimum and average values of the frequencies and corresponding amplitudes of the spectral peaks for normal persons

\begin{tabular}{|c|c|c|c|c|c|c|}
\hline \multirow{2}{*}{$\begin{array}{c}\text { Normal } \\
\text { persons }\end{array}$} & \multicolumn{3}{|c|}{ Amplitude of spectral peak } & \multicolumn{3}{c|}{ Frequency of spectral peak } \\
\cline { 2 - 7 } & Maximum & Minimum & Average & Maximum & Minimum & Average \\
\hline 1 & $0.4154 \times 10^{5}$ & $0.23582 \times 10^{5}$ & $0.30356 \times 10^{5}$ & 257.4292 & 40.0445 & 107.0906 \\
\hline 2 & $0.5185 \times 10^{5}$ & $0.18621 \times 10^{5}$ & $0.37576 \times 10^{5}$ & 160.1782 & 0 & 78.9450 \\
\hline 3 & $0.3613 \times 10^{5}$ & $0.16801 \times 10^{5}$ & $0.24577 \times 10^{5}$ & 97.2510 & 0 & 36.1545 \\
\hline 4 & $0.7041 \times 10^{5}$ & $0.37541 \times 10^{5}$ & $0.54207 \times 10^{5}$ & 171.6195 & 11.4413 & 91.5304 \\
\hline 5 & $1.4237 \times 10^{5}$ & $0.19048 \times 10^{5}$ & $0.56231 \times 10^{5}$ & 148.7369 & 0 & 34.3239 \\
\hline 6 & $0.4283 \times 10^{5}$ & $0.22630 \times 10^{5}$ & $0.30195 \times 10^{5}$ & 245.9879 & 28.6032 & 100.2258 \\
\hline
\end{tabular}

Table 2. Maximum, minimum and average values of the frequencies and corresponding amplitudes of the spectral peaks for the ALS patients

\begin{tabular}{|c|c|c|c|c|c|c|}
\hline \multirow{2}{*}{$\begin{array}{c}\text { ALS } \\
\text { patients }\end{array}$} & \multicolumn{3}{|c|}{ Amplitude of spectral peak } & \multicolumn{3}{c|}{ Frequency of spectral peak } \\
\cline { 2 - 7 } & Maximum & Minimum & Average & Maximum & Minimum & Average \\
\hline 1 & $0.0760 \times 10^{6}$ & $0.31793 \times 10^{5}$ & $0.5615 \times 10^{5}$ & 251.7086 & 17.1619 & 172.5348 \\
\hline 2 & $0.2243 \times 10^{6}$ & $0.50187 \times 10^{5}$ & $0.7012 \times 10^{5}$ & 177.3401 & 0 & 97.9375 \\
\hline 3 & $0.1530 \times 10^{6}$ & $0.42769 \times 10^{5}$ & $0.7263 \times 10^{5}$ & 200.2227 & 0 & 75.0549 \\
\hline 4 & $0.1064 \times 10^{6}$ & $0.36374 \times 10^{5}$ & $0.6486 \times 10^{5}$ & 80.0891 & 0 & 10.7548 \\
\hline 5 & $0.8864 \times 10^{6}$ & $0.29999 \times 10^{5}$ & $1.7667 \times 10^{5}$ & 11.4413 & 0 & 1.8306 \\
\hline 6 & $0.0848 \times 10^{6}$ & $0.42084 \times 10^{5}$ & $0.6111 \times 10^{5}$ & 234.5466 & 0 & 119.6760 \\
\hline
\end{tabular}

\subsubsection{Mean Frequency}

The frequency locations and amplitude values of the peaks of the magnitude spectra of the EMG signals at different frames have been carefully investigated. It is found that these values exhibit significant variation at different frames of the EMG data both in case of normal persons and ALS patients. In order to visualize the level of variations among different frames of a particular person, in Table 1, maximum, minimum and average values of both the frequency and amplitude of the spectral peaks for different normal persons are shown. In a similar fashion, in Table 2, maximum, minimum and average values of both the frequency and amplitude of the spectral peaks for different ALS patients are shown. It can be observed that none of these parameters are consistently distinguishable. As an alternate, in this paper, we propose to utilize mean and median frequencies as spectral features. Considering the product of the frequency and corresponding amplitude at each frequency points of the magnitude spectrum, the mean frequency is computed by taking the average of all such products throughout the entire spectrum. Since in this case both frequency and amplitude values have been given equal weights and all frequencies in the range are considered, a better feature consistency is thus expected.

\subsubsection{Autocorrelation}

The cross-correlation between two signals is a measure of dependency of these two signals on each other. Higher the dependency, larger will be the cross-correlation value. When the two signals involved in the cross-correlation operation become exactly same, the operation is then termed as autocorrelation. In fact, an autocorrelation sequence reflects the degree of similarity at different portions of a time series data. Hence it is a well known operation for measuring the 
Signal \& Image Processing : An International Journal (SIPIJ) Vol.3, No.2, April 2012

hidden periodicity of a signal [21]. In this paper, the characteristics of the autocorrelation function of different frames of EMG data have been investigated.

\section{Autocorrelation of $\mathbf{5}$ Arbitrary Frames of $\mathbf{3}$ Normal Persons}
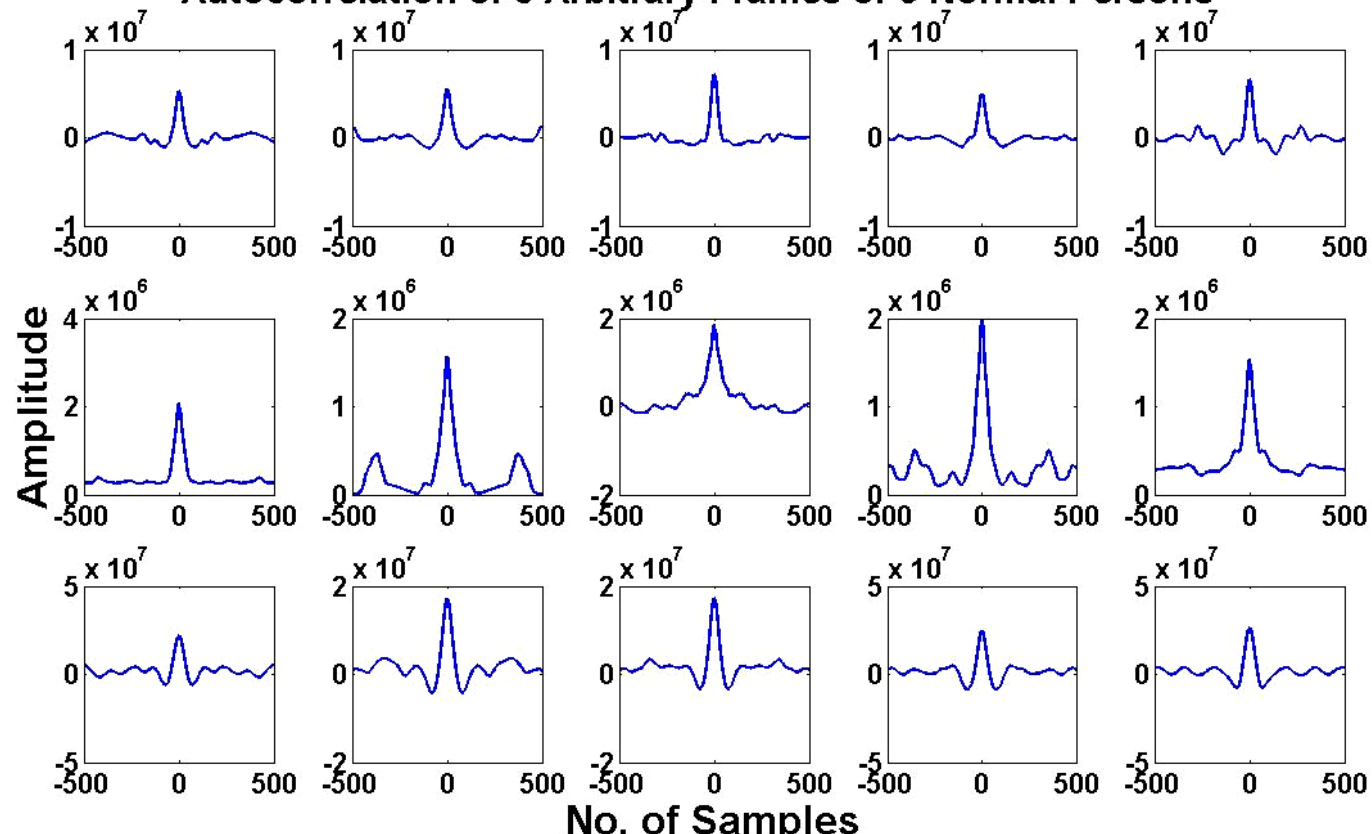

Figure 5. Autocorrelation sequence of the frame of EMG data for the first three normal persons.

Autocorrelation of $\mathbf{5}$ Arbitrary Frames of $\mathbf{3}$ Normal Persons
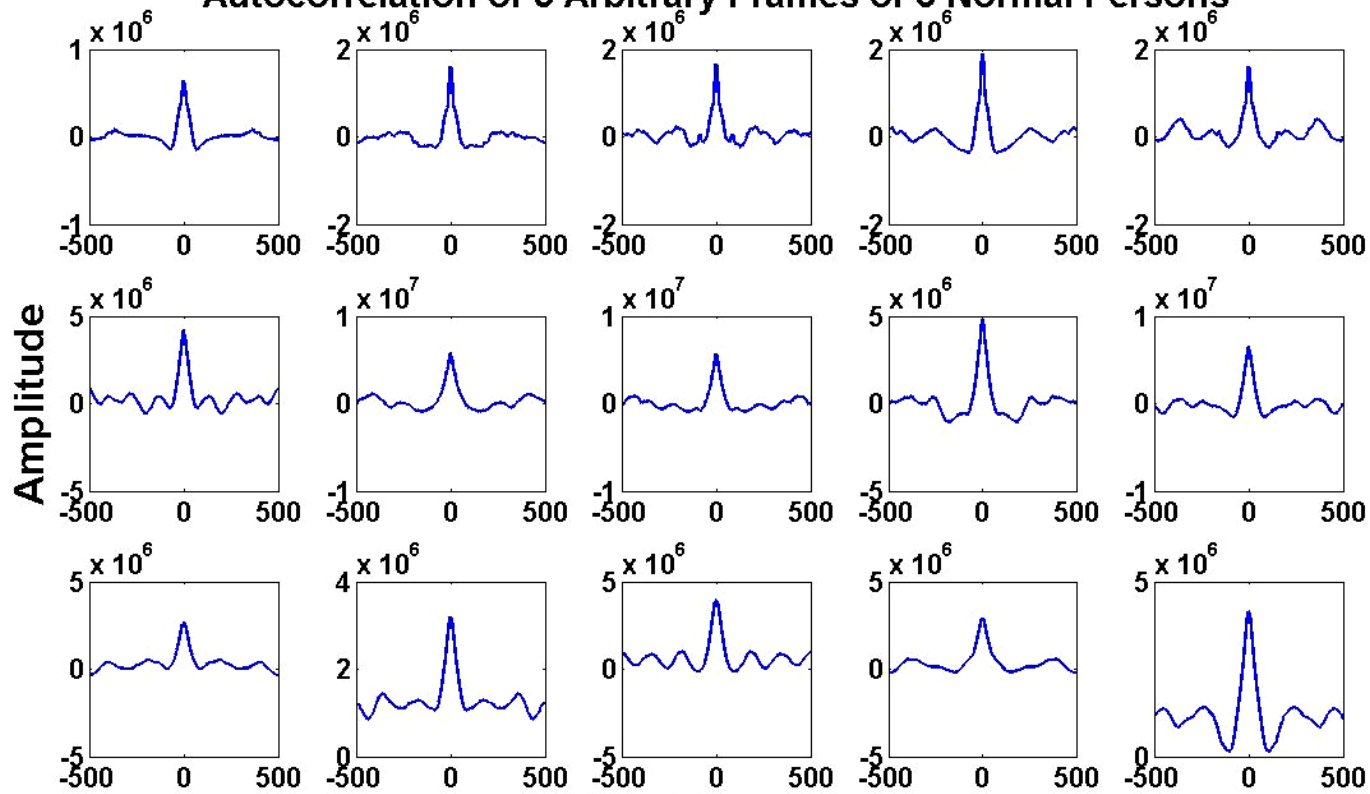

No. of Samples

Figure 6. Autocorrelation sequence of the frame of EMG data for the last three normal persons. 
Signal \& Image Processing : An International Journal (SIPIJ) Vol.3, No.2, April 2012

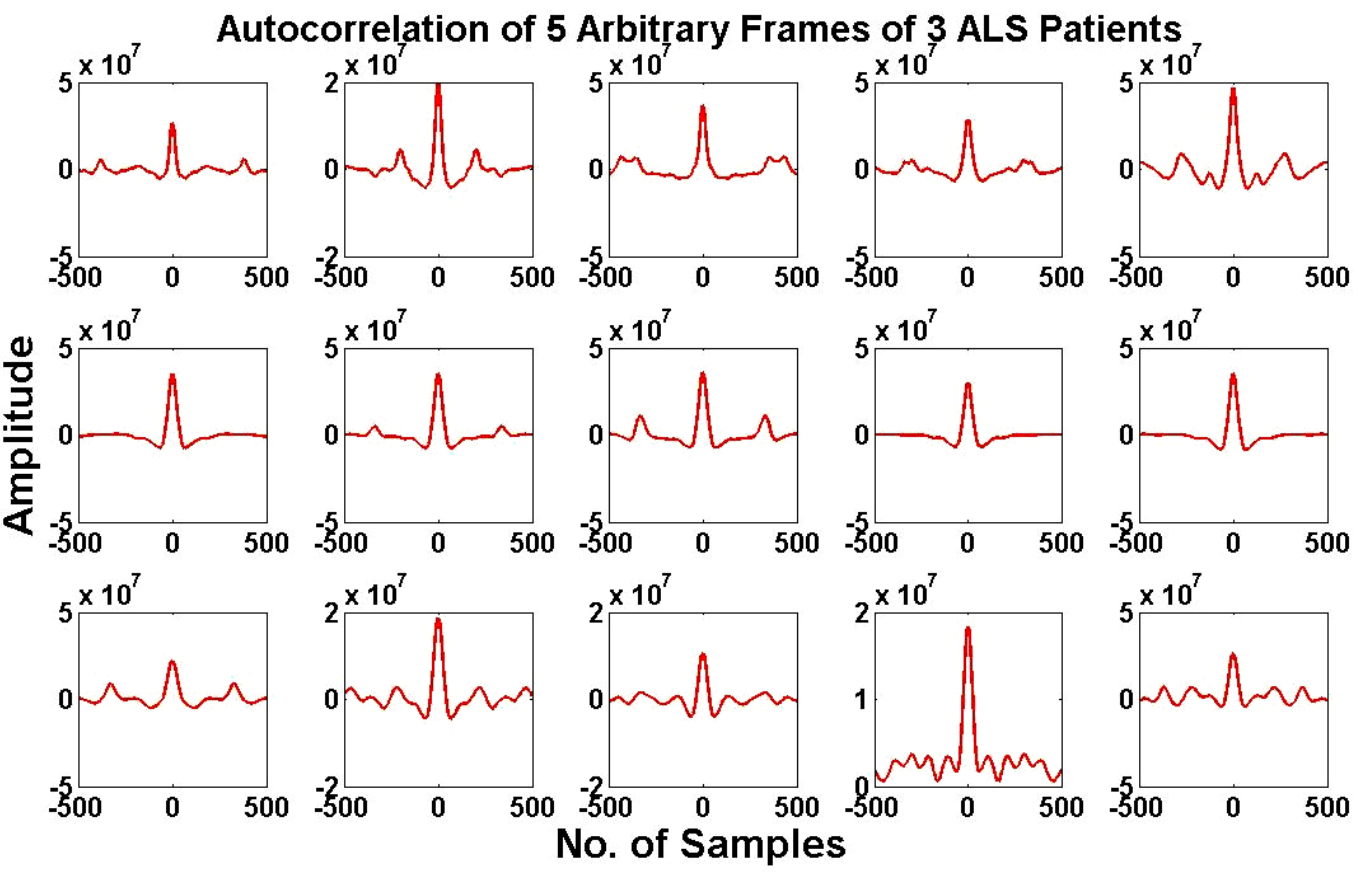

Figure 7. Autocorrelation sequence of the frame of EMG data for the first three ALS patients.

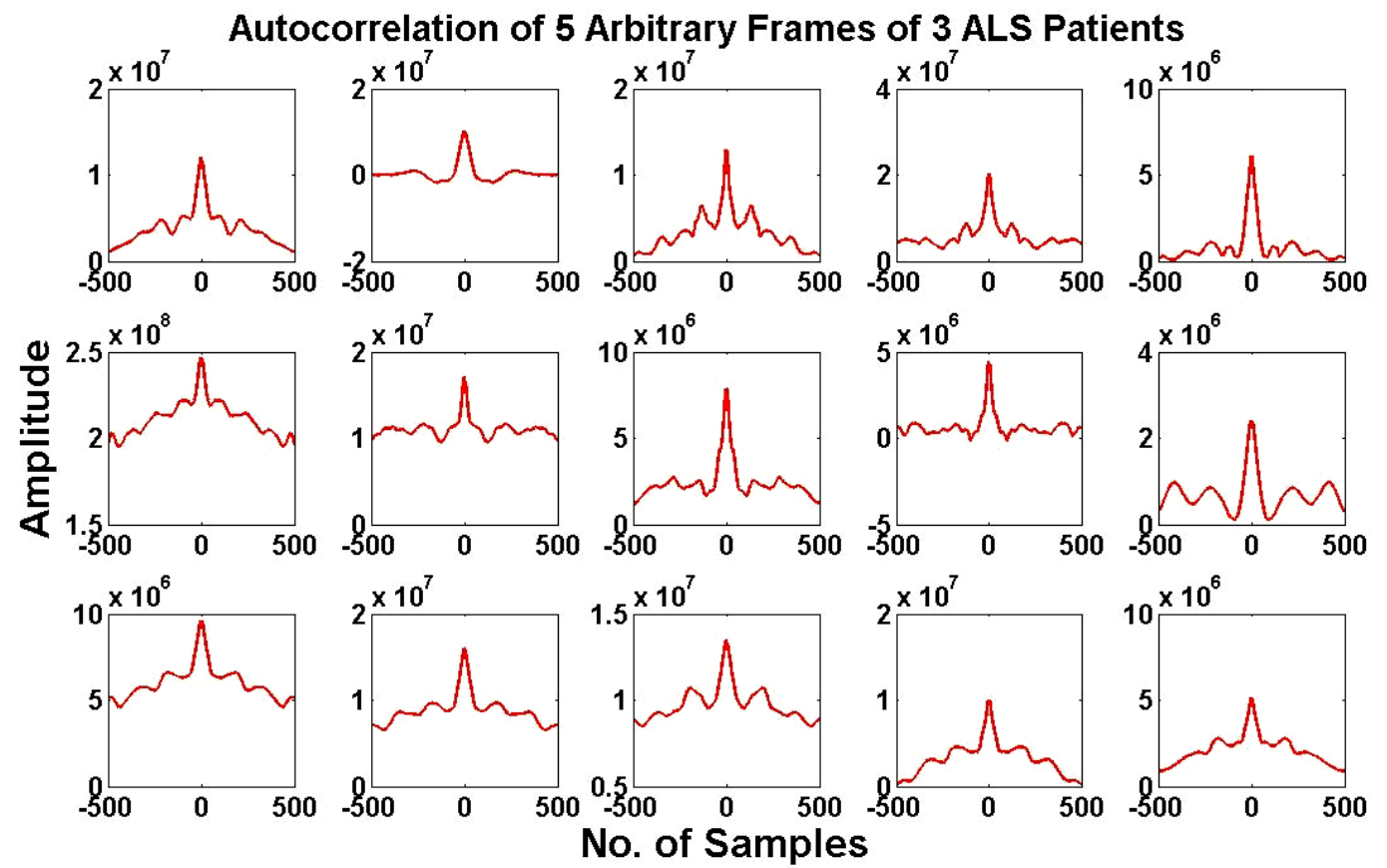

Figure 8. Autocorrelation sequence of the frame of EMG data for the last three ALS patients.

For an N-length sequence of EMG data $f(n)$, its autocorrelation function $r_{f}(\tau)$ can be computed as

$$
r_{f}(\tau)=\frac{1}{N} \sum_{n=0}^{N-1-|\tau|} f(n) f(n+|\tau|)
$$


where $\tau$ denotes the correlation lag. In Figs. 5 and 6, the autocorrelation sequence of the frame of EMG data for the first and last three normal persons are shown, respectively. For each person, five different frames are chosen arbitrarily. In a similar fashion, in Figs. 7 and 8, the autocorrelation sequence of the frame of EMG data for the first and last three ALS patients are shown, respectively.

It can be inferred from these figures that no consistent information regarding the hidden periodicity of the EMG signal is readily observable from the autocorrelation sequence. However, a major distinguishable feature observed in these figures is the overall shape of the correlation functions in case of the normal persons and the ALS patients. In case of the normal persons, the magnitude of the correlation sequence drastically falls right after the zero lag and remains very small at all lags located further from the zero lag. On the contrary, the magnitude of the correlation sequence decreases comparatively at a slower rate and it possesses more strength over the entire lags of the autocorrelation function. Magnitude of the zero lag of the autocorrelation function is taken as a feature in this research.

\subsubsection{Zero-crossing rate (ZCR)}

The Zero-crossing rate (ZCR) expresses the number of times a signal crosses the axis of abscissas. It can be defined as

$$
Z C R=\frac{1}{2 N}\left\{\sum_{k=1}^{N-1}|\operatorname{sgn}[x(k)]-\operatorname{sgn}[x(k-1)]|\right\}
$$

where

$$
\operatorname{sgn}[x]=\left\{\begin{array}{r}
1, x \geq 0 \\
-1, x \leq 0
\end{array}\right.
$$

The random temporal fluctuations of the EMG signal may serve as distinguishable feature. Hence, the ZCR is also considered as a distinguishable feature to comment on the detection of diseases.

\subsubsection{KNN classification}

In pattern recognition, the k-nearest neighborhood algorithm $(\mathrm{KNN})$ is one of the most reliable but simple method of classifying objects based on closest training examples in the feature space. $\mathrm{KNN}$ is a type of instance-based learning or lazy learning where the function is only approximated locally and all computations are deferred until the classification. In this paper, for the classification of the EMG data into two classes based on the time and frequency domain features, the KNN classifier is employed.

\section{EMG Signal ANALYSIS AND Feature EXTRACTION}

For the purpose of detecting the ALS patients from the given EMG data, 18 datasets of 6 normal persons and 18 datasets of 6 ALS patients are used. Each EMG dataset has a total number of 262,134 samples at a rate of 23,438 samples per second. Thus, each of these single channel datasets has total time duration of $11.184 \mathrm{sec}$. 
Signal \& Image Processing : An International Journal (SIPIJ) Vol.3, No.2, April 2012
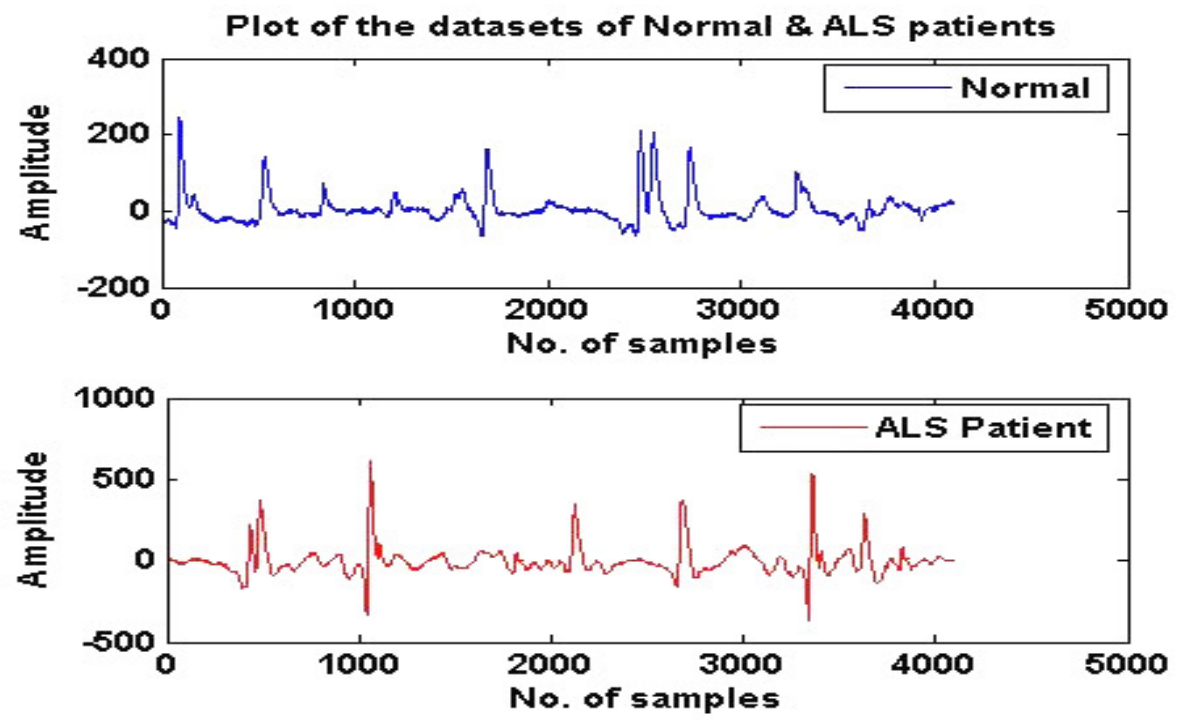

Figure 9. EMG data pattern of a normal person and the ALS patient.

RMS comparison between Normal and ALS patients

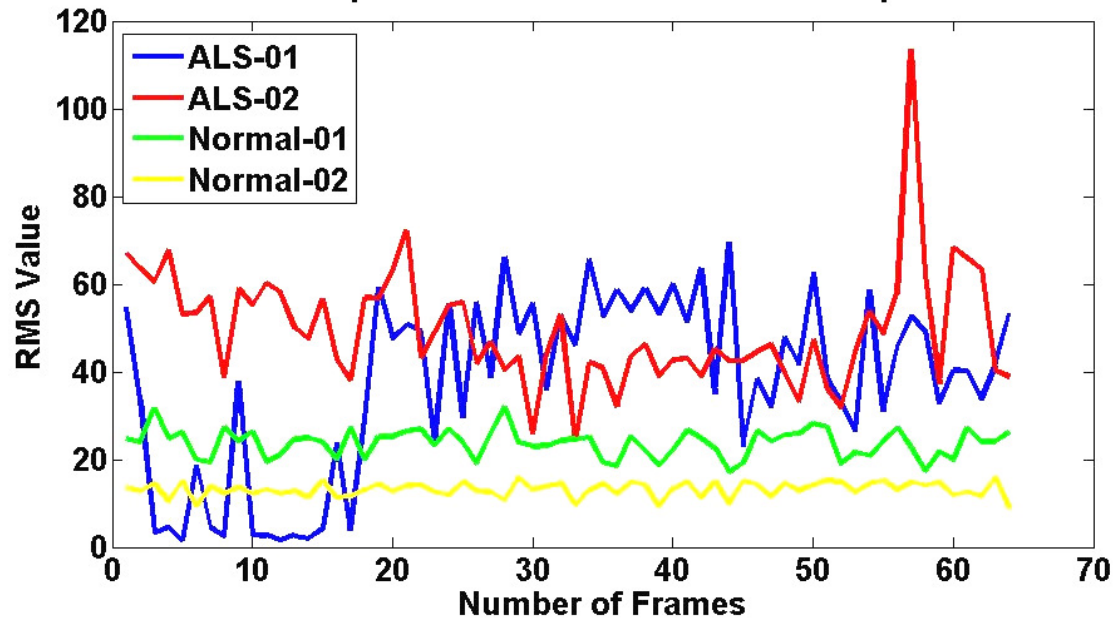

Figure 10. RMS values obtained from each frame of the EMG data considering two normal persons and two ALS patients.

At first, a single dataset is segmented into 64 distinct frames, each consisting of 4096 number of samples. In Fig. 9, the pattern of the EMG data are shown for a normal person and an ALS patient, respectively. Next, the root-mean-square (RMS) value of each frame of data is calculated for both normal and ALS datasets. In Fig. 10, the RMS values obtained from each frame of the EMG data considering two normal persons and two ALS patients are plotted. It is found from the analytical results as well as from Fig. 10 that the RMS values corresponding to the ALS patients fluctuate abruptly in the initial and final frames but exhibit a stable range of values between 30 and 56 in the middle frames of every datasets. On the other hand, RMS values corresponding to a normal person show a steady range of values which does not exceed 28 for all the frames in a dataset. Finally, 25 frames (from $30^{\text {th }}$ frame to $55^{\text {th }}$ frame) are selected out of 64 frames of both the normal persons and ALS patients for further processing to extract different features.

Since the energy of the EMG signal is mostly concentrated in the low frequency regions, a low pass filter is used to reduce the effect of high frequency regions. The low pass filtered EMG signal is then used for feature extraction. Proposed time and frequency domain features, such as 
Signal \& Image Processing : An International Journal (SIPIJ) Vol.3, No.2, April 2012

magnitude spectrum, mean frequency, autocorrelation and ZCR are computed on an individual frame basis. For both the cases of normal persons and the ALS patients, average results obtained from 25 frames of every datasets are considered to construct the feature vector. Finally the KNN classifier is employed to detect the ALS affected EMG signals from the normal signals.

\section{RESULT AND DISCUSSIONS}

In this paper, we propose different time and frequency domain characteristics for the classification of EMG signals to detect the ALS patients and distinguish them from the normal group. It is found that the proposed features, such as the spectral peak level, mean frequency value, zero crossing rate and the value of the zero lag of the autocorrelation function have the capability of distinguishing the EMG data of the ALS patients from that of the normal persons.

In Fig. 11, average amplitude values of spectral peaks of different datasets corresponding to normal persons and the ALS patients are shown. As expected the level of average values of spectral peaks corresponding to the ALS patients is much higher than that of corresponding to normal persons. In Fig. 12, average zero lag values of the autocorrelation function of different datasets corresponding to normal persons and the ALS patients are shown. Here also a similar distinguishable behaviour is observed between the normal persons and the ALS patients. It is clearly observed from Figs. 11 and 12 that the proposed features offer a high degree of separability between the two classes, which ensures a better classification accuracy.

In Fig. 13, the average ZCR values of EMG signals of different datasets corresponding to normal persons and the ALS patients are shown. Unlike the previous two cases, in this case the degree of separability is not much satisfactory. In Fig. 14, the average mean frequency values of the magnitude spectrum of different datasets corresponding to normal persons and the ALS patients. Here also a moderate degree of separability is obtained. Hence, it is expected that in comparison the last two features, namely the ZCR and mean frequency, the first two features based on the spectral peak and autocorrelation function may provide better classification performance.

In order to show the classification performance, each feature has been tested using the KNN classifier. The most widely used leave-one-out cross validation algorithm is utilized for the testing purpose. In this case, among several datasets only one dataset is taken away at a time for the purpose of testing against the remaining all datasets. Excluding the test dataset, remaining datasets are used for the training of the classifier. Depending on the classifier output value as defined in the group parameter of the classifier, the EMG signals are classified as normal or ALS affected EMG signals. Some statistical performance measures, such as specificity, sensitivity and accuracy are computed to investigate the clsssification performance. These statistical performance measures are defined as follows:

Specificity: Number of correctly classified normal subjects/number of total normal subjects.

Sensitivity: Number of correctly classified ALS subjects/number of total ALS subjects.

Classification accuracy: Number of correctly classified subjects/number of total subjects.

Table 3 gives the overall prformance measurement of the clssifier for the 4 features individually. As expected, the highest success rate of $100 \%$ is obtained for both the proposed autocorreltion and spectral peak based features. It is observed from the table that the features like ZCR and the mean frequency provide comparatively low classification accuracy. 
Signal \& Image Processing : An International Journal (SIPIJ) Vol.3, No.2, April 2012

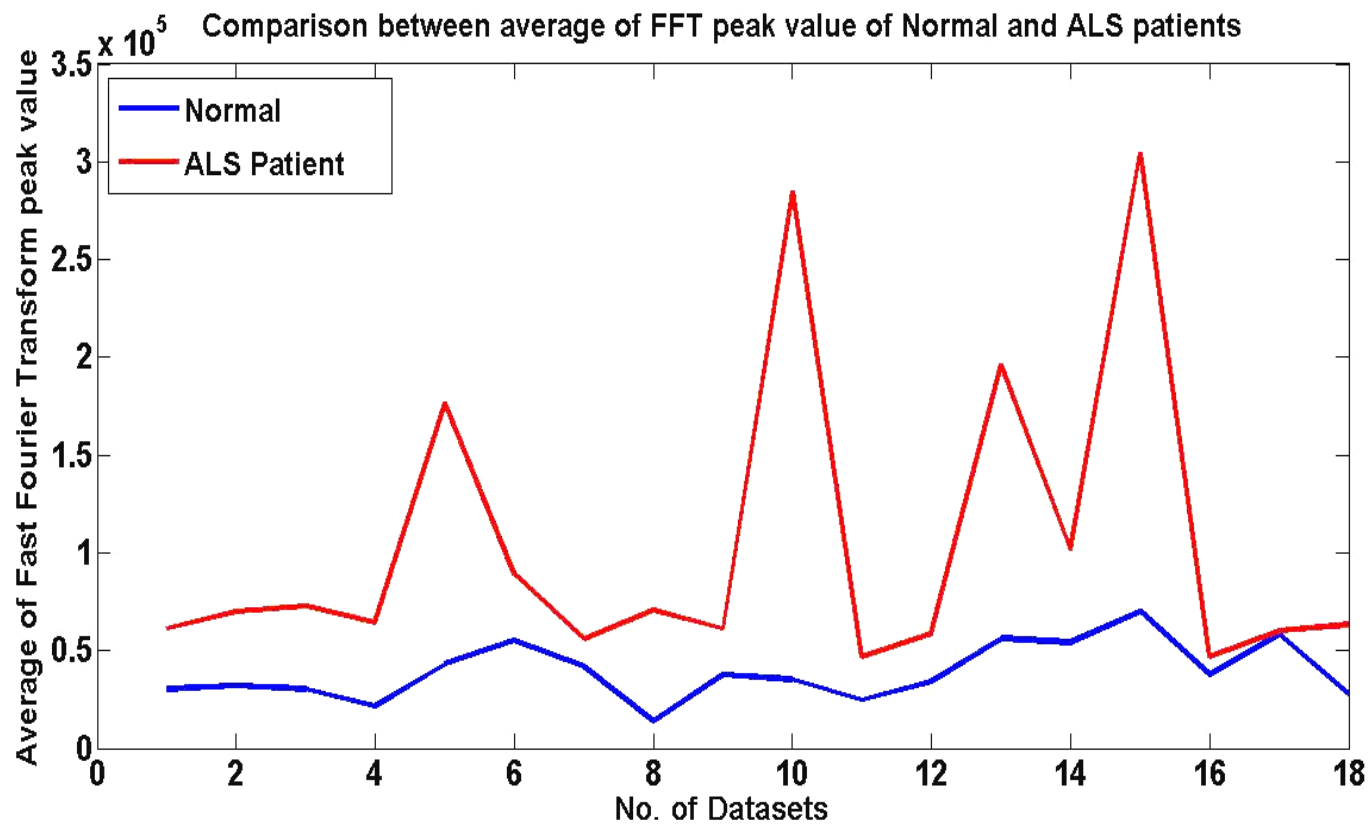

Figure 11. Average amplitude values of spectral peaks of different datasets corresponding to normal persons and the ALS patients.

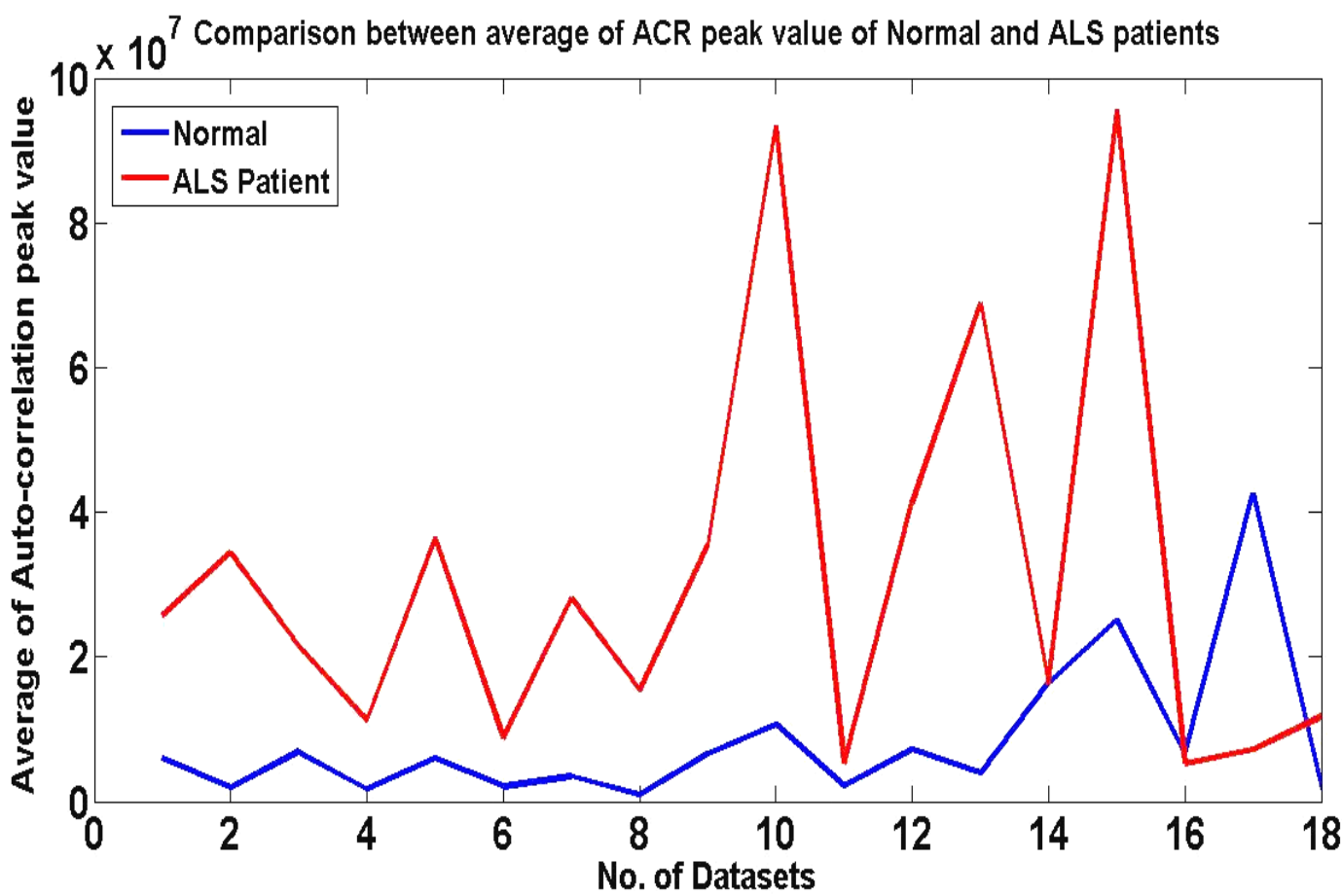

Figure 12. Average zero lag values of the autocorrelation function of different datasets corresponding to normal persons and the ALS patients. 
Signal \& Image Processing : An International Journal (SIPIJ) Vol.3, No.2, April 2012

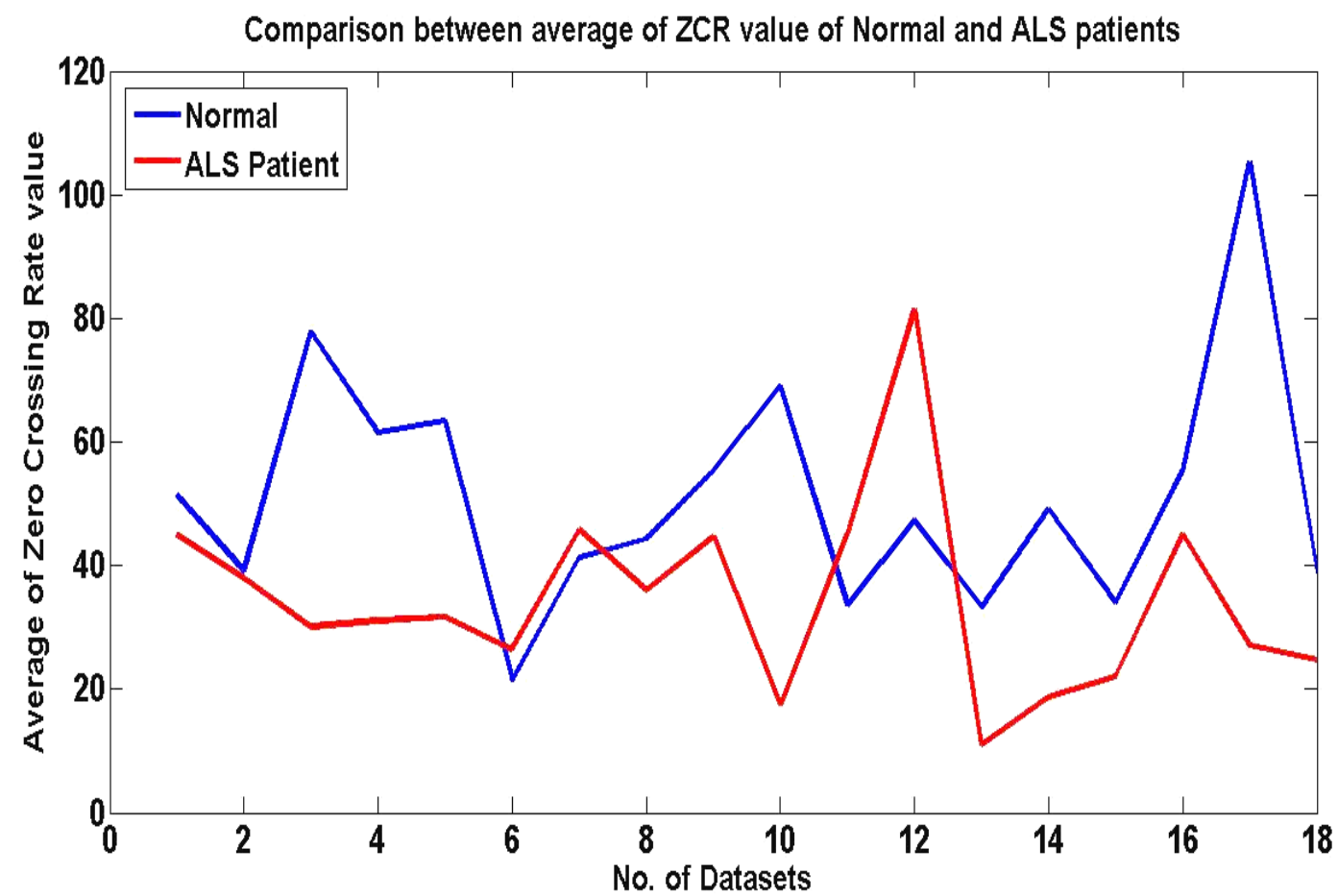

Figure 13. Average ZCR values of EMG signals of different datasets corresponding to normal persons and the ALS patients.

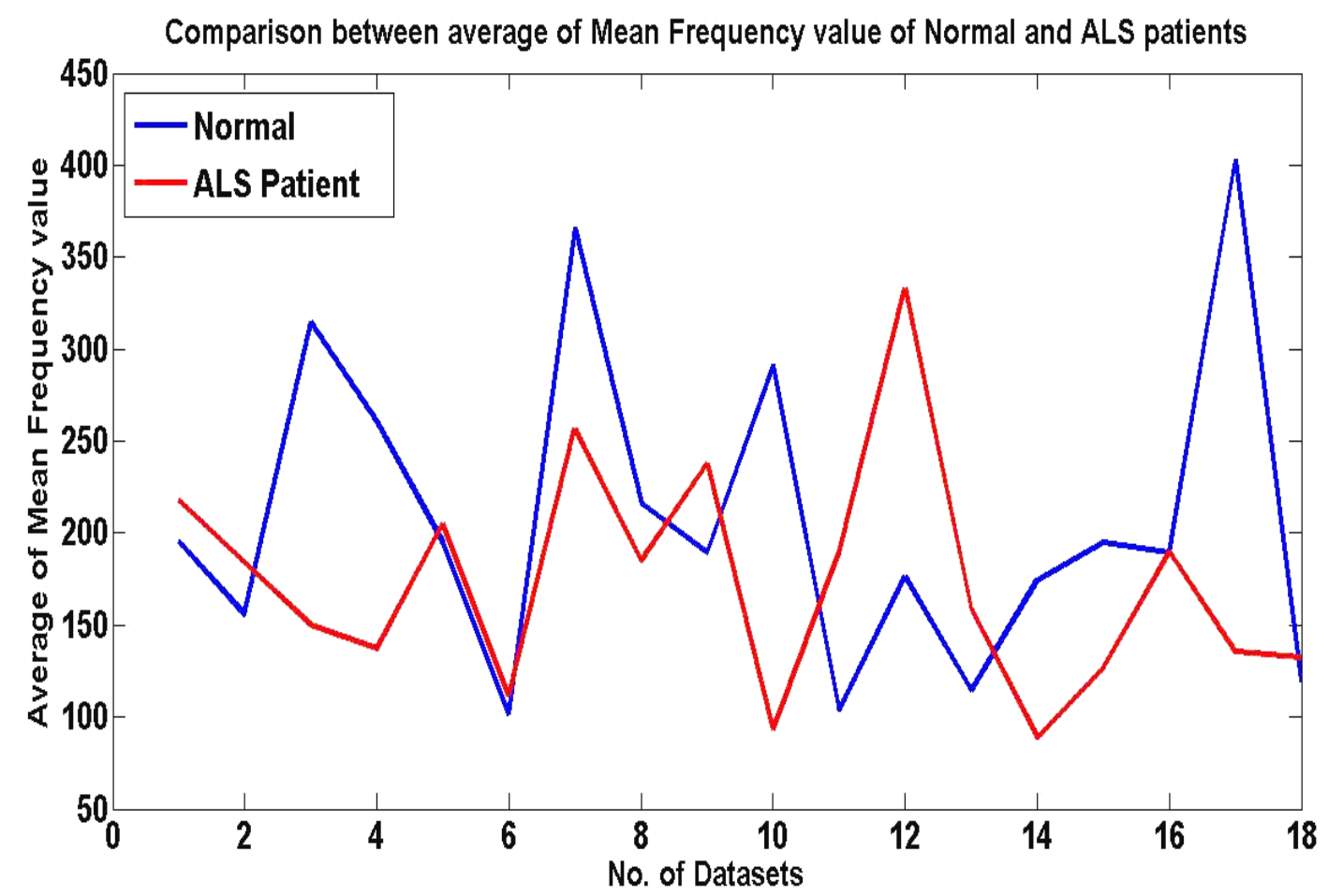

Figure 14. Average mean frequency values of the magnitude spectrum of different datasets corresponding to normal persons and the ALS patients. 
Signal \& Image Processing : An International Journal (SIPIJ) Vol.3, No.2, April 2012

Table 3. Classification performance of the proposed features

\begin{tabular}{|l|c|c|c|}
\hline Feature & Accuracy $(\boldsymbol{\%})$ & Specificity (\%) & Sensitivity (\%) \\
\hline Spectral peak & 100 & 100 & 100 \\
\hline Mean Frequency & 69.5 & 66.7 & 72.2 \\
\hline Autocorrelation & 100 & 100 & 100 \\
\hline ZCR & 72.2 & 72.2 & 72.2 \\
\hline
\end{tabular}

\section{CONCLUSION}

A comprehensive analysis of time and frequency domain features of EMG signals is presented in this paper with an objective to develop an efficient classification scheme to handle the two class problem of separating the EMG signals of normal control group and the ALS patients. It is shown that proper feature selection can provide an excellent classification performance even for a very complicated biomedical signal like EMG. Among the proposed spectral features, the average values of spectral peak exhibits better performance in comparison to the mean frequency. On the other hand, among the proposed time domain features, average zero lag values of the autocorrelation function offers better classification performance than the most common ZCR feature. The main reason behind the superiority of the classification performance obtained by using the proposed two features is the high degree of inter-class feature separability. Because of the robustness of the proposed features, even use of a simple KNN classifier can result in $100 \%$ classification accuracy for the case of spectral peak and autocorrelation based features.

\section{REFERENCES}

[1] J. V. Basmajian and C. J. De Luca, Muscles Alive. Their Function Revealed by Electromyography. Baltimore: Williams \& Wilkens, 1985.

[2] M. Akay, Biomedical signal processing, 1 ed. Sandiego, California: Academic Press, Inc, 1994.

[3] D. Stashuk, "EMG signal decomposition: how it is performed and how it can be used?," Journal of Electromyography and Kinesiology, vol. 11, pp. 151-173, 2001.

[4] M. B. I. Reaz, M. S. Hussain and F. M. Yasin, "Techniques of EMG signal analysis: detection, processing, classification and applications," Biological Procedures Online, vol. 8, no. 1, pp. 11-35, 2006.

[5] S. Shahid, "Higher Order Statistics Techniques Applied to EMG Signal Analysis and Characterization," PhD Thesis, University of Limerick, Ireland, 2004.

[6] R. F. M. Kleissen, J. H. Buurke, J. Harlaar and G. Zilvold, "Electromyography in the biomechanical analysis of human movement and its clinical application," Gait Posture, vol. 8, no. 2, pp. 143-158, 1998.

[7] J. R. Cram, G. S. Kasman and J. Holtz, "Introduction to Surface Electromyography," Aspen Publishers Inc, Gaithersburg, Maryland, 1998.

[8] A. Merlo and D. Farina, "A Fast and Reliable Technique for Muscle Activity Detection from Surface EMG Signals,” IEEE Trans. Biomed. Eng., vol. 50, pp. 316 - 323, 2003.

[9] P. Guglielminotti and R. Merletti, "Effect of electrode location on surface myoelectric signal variables: a simulation study," 9th Int. Conf of ISEK, Florence, Italy, 1992. 
Signal \& Image Processing : An International Journal (SIPIJ) Vol.3, No.2, April 2012

[10] National Institute of Neurological Disorders and Stroke (NINDS). "ALS (Amyotrophic Lateral Sclerosis) Fact Sheet," NIH Publication No. 10-916, 2010. Available: http://www.ninds.nih.gov/disorders/amyotrophiclateralsclerosis/detail_ALS.htm.

[11] P. K. Kasi, "Characterization of Motor Unit Discharge Rate in Patients with Amytrophic Lateral Sclerosis (ALS)," Worcester Polytechnic Institute, May 2009.

[12] P. N. Leigh and A. Al-Chalabi, "Recent advances in amyotrophic lateral sclerosis," Curr. Opin. Neurol., vol. 13, pp. 397-405, 2000.

[13] M. Behnia and J. Kelly, "Role of electromyography in amyotrophic lateral sclerosis. Muscle Nerve," vol. 14, pp. 1236-1241, 1991.

[14] E. H. Lambert and D. W. Mulder, "Electromyographic studies in amyotrophic lateral sclerosis," Mayo Clin. Proc., vol. 32, pp. 441-446, 1957.

[15] E. H. Lambert, "Electromyography in amyotrophic lateral sclerosis," pp. 135-153. In: F.H. Norris, L.T. Kurland (Eds.) "Motor neuron diseases: research in amyotrophic lateral sclerosis and related disorders," New York, Grune and Stratton, 1969.

[16] M. Nikolic, "Detailed Analysis of Clinical Electromyography Signals EMG Decomposition, Findings and Firing Pattern Analysis in Controls and Patients with Myopathy and Amytrophic Lateral Sclerosis", PhD Thesis, Faculty of Health Science, University of Copenhagen, 2001.

[17] L. Cohen, “Time-frequency analysis,” Englewood Cliffs, Prentice-Hall; New Jersey, USA, 1995.

[18] M. Amin, L. Cohen and W. J. Williams, "Methods and Applications for Time Frequency Analysis," Conference Notes, University of Michigan, 1993.

[19] F. G. Nihal and K. Sabri, "Classification of EMG Signals Using PCA and FFT," Journal of Medical Systems, vol. 29, no. 3, 2005.

[20] A. O. Andrade, P. J. Kyberd and S. D. Taffler, "A novel spectral representation of electromyographic signals," in: R.S. Leder (Ed.), Engineering in Medicine and Biology Society - 25th Annual International Conf, vol. 1, IEEE, Cancun, Mexico, pp. 2598-2601, 2003.

[21] A. F. Kohn, "Autocorrelation and Cross-correlation Methods," Wiley Encyclopedia of Biomedical Engineering, Ed. Metin Akay, New Jersey: John Wiley \& Sons, pp. 23, 2006.

\section{Authors}

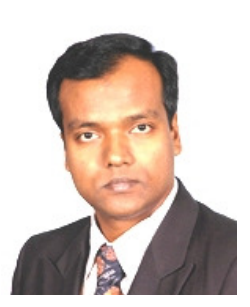

Shaikh Anowarul Fattah received the B.Sc. and M.Sc. degrees from Bangladesh University of Engineering and Technology (BUET), Dhaka, Bangladesh, in 1999 and 2002, respectively, both in electrical and electronic engineering (EEE). He received the Ph.D. degree in electrical and computer engineering from Concordia University, Montreal, QC, Canada in 2008. He was a visiting Postdoctoral Fellow in the Department of Electrical Engineering at Princeton university, New Jersey, USA. He joined as a Lecturer in Jan. 2000 in the Department of EEE, BUET where he is currently serving as an Associate Professor.

He is a recipient of the Dr. Rashid Gold Medal for the best academic performance in M.Sc. He was selected as one of the Great Grads of Concordia University in 2008-2009 and the winner of Concordia University's the 2009 Distinguished Doctoral Dissertation Prize in Engineering and Natural Sciences. During his Ph.D. program, he has also received Concordia University Graduate Fellowship, Power Corporation of Canada Graduate Fellowship, a New Millennium Graduate Scholarship, Hydro Quebec Awards, International Tuition-fee Remission Award, and Doctoral Teaching Assistantship. He is the recipient of URSI Canadian Young Scientist Award 2007 and was the first prize winner in the SYTACOM Research Workshop in 2008. Many projects and theses under his supervision have been awarded First and Second prizes and Honorable Mentions in Concordia University and BUET. He was a recipient of NSERC Postdoctoral Fellowship in 2008 . 
Signal \& Image Processing : An International Journal (SIPIJ) Vol.3, No.2, April 2012

Dr. Fattah has published more than eighty international journal and conference papers. His research interests include the areas of system identification and modeling, speech, audio, and music signal processing, genomic signal processing, biomedical signal processing, biometric recognition for security, multimedia communication and control system. He is serving as technical committee members of different international conferences. He served as a member secretary of the EUProW 2009 and 2011. He was awarded the Best Organizer Award 2011 from the Department of EEE, BUET. He is the Technical Chair of APMEE 2012 and Organizing Secretary of ICECE 2012. He is the reviewer of different international and IEEE journals, such as IEEE Transactions on Circuits and Systems and IEEE Transactions on Signal Processing. Presently, he is the Vice-Chair of the IEEE, Bangladesh Section. Dr. Fattah was Co-editor and now is the Editor of Journal of Electrical Engineering, Institute of Engineers, Bangladesh..

Md Asif Iqbal was born in Narayangonj, Bangladesh on June 02, 1988. He received his B.Sc. degree from Military Institute of Science \& Technology (MIST), Dhaka, Bangladesh in January 2010, from the department of Electrical, Electronic \& Communication Engineering (EECE). He is continuing his M.Sc. in Electronics and Communication Engineering in Bangladesh University of Engineering \& Technology (BUET), Dhaka, Bangladesh. He is presently working as a lecturer in the department of EECE, MIST.

Marzuka Ahmed Jumana was born in Dhaka, Bangladesh on January 29, 1989. She received her B.Sc. degree from Military Institute of Science \& Technology (MIST), Dhaka, Bangladesh in December,2010, from the department of Electrical, Electronic \& Communication Engineering (EECE). She is continuing her M.Sc in Electronics and Communication Engineering in Bangladesh University of Engineering \& Technology (BUET), Dhaka, Bangladesh. She is presently working as a lecturer in the department of EECE, MIST.

A.B.M. Sayeed Ud Doulah was born in Dhaka, Bangladesh on October 02, 1988. He obtained his B.Sc. degree from Military Institute of Science \& Technology (MIST), Dhaka, Bangladesh in December, 2010, from the department of Electrical, Electronic \& Communication Engineering (EECE). He is pursuing his M.Sc. in Electronics and Communication Engineering in Bangladesh University of Engineering \& Technology (BUET), Dhaka, Bangladesh. His research interest includes biomedical signal processing, Image processing, speech signal processing, nano biomedical etc. He is presently working as a lecturer in the department of EECE, MIST. 